\title{
Exploring the utility and effectiveness of tidal stream energy resource assessment and characterisation standards: A case study
}

\author{
V. Ramos \\ Centre for Ocean Energy Research, Maynooth University, Maynooth, Ireland \\ R. Carballo \\ Hydraulic Engineering, University of Santiago de Compostela, EPS, Lugo, Spain \\ John V. Ringwood \\ Centre for Ocean Energy Research, Maynooth University, Maynooth, Ireland
}

\begin{abstract}
For tidal stream energy to become a fully-fledged renewable energy source, a complete understanding of the tidal stream resource characterisation is required. For this purpose, the International Electrotechnical Commission (IEC) has elaborated a technical specification for the assessment of the tidal stream resource, "IEC-TS 62600-201: Marine energy-Wave, tidal and other water current converters - Part 201: Tidal energy resource assessment and characterisation" (IEC-62600-201), which presents a wide set of recommendations with the aim of standardising tidal stream resource characterisation. The IEC-62600-201 divides resource assessments into two different classes: feasibility and layout design. The model implementation (mesh resolution, boundary conditions) and the effort required in terms of computational time varies significantly from one class to another. For these reasons, the objective of the present work is to explore the IEC-62600-201 using the Orkney Region (N Scotland) as a case study. Overall, it was found that the IEC-62600-201 works well, offering a detailed characterisation of the resource; however, in order to make the IEC-62600-201 more manageable some aspects such as the grid resolution requirements and the TEC modelling should be revisited for future editions.
\end{abstract}

\section{INTRODUCTION}

As a result of different policies, which aim to increase the importance of renewable energy sources in the energy mix (Rourke, Boyle, \& Reynolds 2009), marine energies have attracted great attention due to its large energy potential (Bahaj 2011). Among them is tidal stream energy, which taps the kinetic energy of the currents caused by the tide. Tidal stream power presents significant advantages in comparison with other sources of renewable energy: (i) the resource can be predicted in advance thanks to the astronomical nature of the driving force; (ii) the load factor is comparatively high due to the properties of the fluid; and (iii) land occupation is minimal (Carballo, Iglesias, \& Castro 2009, Fergal O. Rourke, Fergal Boyle, \& Anthony Reynolds 2010).

This growing interest in tidal stream energy has translated into a large number of tidal resource assessment studies all over the world (Carballo, Iglesias, \& Castro 2009, Jinhai Zheng, Peng Dai, \& Jisheng Zhang 2015), which have been mainly carried out by means of hydrodynamic numerical models validated against field measurements.
However, the approaches used vary significantly for different aspects such as the time scale of the assessments, the inclusion or not of the effects derived from the wave-current interactions and the consideration or not of the potential variations in the magnitude of the tidal resource due to the presence and operation of the Tidal Energy Converters (TECs) (Hashemi, Neill, Robins, Davies, \& Lewis 2015).

In an attempt to standardise the approach to resource assessment, the International Electrotechnical Commission (IEC) has recently put forwards a set of recommendations to develop a uniform methodology with the aim of ensuring consistency and accuracy in tidal resource assessment: "IEC-TS 62600-201: Marine energy - Wave, tidal and other water current converters-Part 201: Tidal energy resource assessment and characterisation" (from now on referred as IEC-62600-201) (International Electrotechnical Commision, IEC 2014). The IEC62600-201 classifies the tidal resource assessments into two different categories, feasibility and layout design, denoted as Stage 1 and 2, respectively. Stage 1 assessments are intended to obtain a first approximation of the tidal stream energy resource 
over a relatively large area (i.e. estuary or channel) with a medium level of uncertainty. Stage 2 assessments are used to obtain a detailed characterisation in a specific area to determine the TEC Annual Energy Production (TAEP) with a low degree of uncertainty. In addition, IEC-62600-201 also offers a detailed series of recommendations regarding the procedure to follow in the fields of data collection, numerical modelling, data analysis and reporting of the results. Furthermore, the numerical setup varies considerably from Stage 1 to Stage 2 in terms of boundary conditions, spatial resolution and physical processes to be considered, which has a significant impact on the level of effort required in the modelling process.

Against the foregoing backdrop, this work aims to focus on: (i) The evaluation of the two different stages of resource assessment proposed by the IEC-62600-201 in terms of uncertainty of the estimated TAEP and the effort required in the model setup and application with special emphasis on the computational demands and (ii) the provision of feedback into IEC-62600-201 with the aim of offering practical recommendations to the users and future edits to be considered during the maintenance of IEC-62600-201.

This paper is structured as follows: Section 2 presents the main characteristics of IEC-62600-201. Sections 3, 4 and 5 show the main characteristics of the case study used in this work. Section 6 shows the results obtained for the validation of the hydrodynamic model and tidal resource characterisation. Section 7 presents a discussion with respect to the main characteristics of IEC-62600-201. Finally, in Section 8 , conclusions are drawn.

\section{IEC 62600-201 TS: MARINE}

ENERGY - WAVE, TIDAL AND

OTHER WATER CURRENT

CONVERTERS-PART 201: TIDAL ENERGY RESOURCE ASSESSMENT AND CHARACTERISATION

The main goal of IEC-62600-201 is to provide a uniform methodology in order to ensure consistency and accuracy in the modelling, measurement, characterisation and analysis of the tidal stream resource with the aim of estimating accurately the TEC Annual Energy Production (TAEP) at locations suitable for the installation of a tidal farm. In this section only the main characteristics of modelling aspects of IEC-62600-201 will be presented. For details of other aspects, such as data measurement and data analysis, the readers are referred to the IEC-62600-201 document (International Electrotechnical Commission, IEC 2014).

As mentioned in the previous section, the technical specification classifies the tidal resource assessments into two different classes: feasibility and layout design, which are denoted as Stage 1 and 2 , respectively. The main characteristics of each class are summarised in Table 1 .

As can be observed in Table 1, Stage 1 studies are focused on investigating the tidal resource over a particular area of study such as an estuary or a channel with the aim of assessing the viability for the installation of a tidal farm. On the other hand, Stage 2 studies are focused on generating detailed and accurate information on the energy resource to determine the most ideal location and typology of TEC deployments, as well as their power production. The decrease in the level of uncertainty from Stage 1 to Stage 2 may result from different refinement procedures such as: (i) measurements and/or modelling over longer periods; (ii) availability of additional, and or higher quality measurements; (iii) finer discretisation in space and time; (iv) use of improved boundary conditions and (v) improvements in modelling techniques during the evolution of the project.

The model setup requirements may vary considerable from one class to another in terms of (i) physical processes to be considered (wave-current interaction, baroclinic flows, etc...); (ii) boundary conditions (i.e. min number of harmonic constituents required); (iii) spatial resolutions (vertical resolution $2 \mathrm{D}$ vs $3 \mathrm{D}$, min horizontal grid resolution) and (iv) temporal resolution. All these requirements are summarised in Table 2 .

Regardless of the class considered, the main objective is to determine the TAEP, which can be computed according to the following expression:

$$
T A E P=N_{h} \cdot A \cdot \sum_{i=1}^{N_{B}} P_{i}\left(U_{i}\right) \cdot f_{i}\left(U_{i}\right)
$$

where, $N_{h}$ is the number of hours in the simulated year, $A$ is the expected TEC availability, $N_{B}$ represents the total number of velocity bins in the TEC power curve, $P_{i}\left(U_{i}\right)$ describes the power output generated by the $i t h$ velocity bin of the TEC power curve, $U_{i}$ stands for the mean current velocity of the ith bin of the TEC power curve, and $f_{i}\left(U_{i}\right)$ describes the proportion of time for which the mean current velocity falls within the ith velocity bin of the TEC power curve

Depending on the nature of the tidal project, the requirements for the hydrodynamic models to

Table 1. Classes of resource assessment IEC-TS.

\begin{tabular}{llll}
\hline Stage & Aim & Area & $\begin{array}{l}\text { Level of } \\
\text { uncertainty }\end{array}$ \\
\hline Stage 1 & Feasibility & Estuary/Channel & Medium \\
Stage 2 & Layout design & Development site & Low \\
\hline
\end{tabular}


Table 2. IEC-62600-201 model setup recommendations.

\begin{tabular}{lll}
\hline Component & Stage 1 & Stage 2 \\
\hline Modelling & & \\
Num. of harmonic constituents & $4-8$ & $8-12$ \\
Vertical discretisation & $2 \mathrm{D}$ & $3 \mathrm{D}$ \\
Min. grid resolution & $500 \mathrm{~m}$ & $50 \mathrm{~m}$ \\
Period of run & $>35$ days & $>35$ days \\
Energy extraction impacts & $\circ$ & $*$ \\
$\begin{array}{l}\text { Physical data requirement } \\
\text { Wave characteristics }\end{array}$ & $*$ & $*$ \\
Meteorological data & $*$ & $*$ \\
$\begin{array}{l}\text { Flow structure/Eddies/ } \\
\quad \text { Turbulence }\end{array}$ & $*$ & $*$ \\
$\begin{array}{l}\text { Stratification, Seawater density } \\
\text { and sediment measurement }\end{array}$ & $*$ & $*$ \\
\hline
\end{tabular}

- Mandatory * Recommended ○ No mandatory

calculate the TAEP may differ significantly. For instance, in projects in which the total power output is expected to be less than $10 \mathrm{MW}$ and/or the $2 \%$ of the theoretical energy resource, the TAEP may be computed directly from the time series of velocities obtained from a hydrodynamic model validated against field measurements. In addition, for these projects, the TAEP may also be calculated from direct field measurements at the locations of interest. Conversely, for larger projects (i.e. more than $10 \mathrm{MW}$ and/or the $2 \%$ of the theoretical energy resource) IEC-62600-201 does require inclusion of the representation of the TEC operation in the hydrodynamic model to account for the potential effects on the magnitude of tidal flow velocities and, therefore, to represent them on the velocity probability distribution. With the purpose of modelling the TEC operation within a hydrodynamic model, IEC-62600-201 proposes different methodologies that can be found in the literature (Ramos, Carballo, Sanchez, Veigas, \& Iglesias 2014).

Finally, the models must be validated for each class of resource assessment. IEC-62600-201 establishes that the model validation should be achieved by comparing the TAEP results obtained from the field measurements and the computed time-series of velocity at a specific location, using a TEC technology as a reference. For Stage 2 assessments, the comparison should be carried out at different critical depths alongside the water column. In any case, IEC-62600-201 does not set either a minimum number of validation points or a statistical criteria to consider the model successfully validated.

\section{CASE STUDY: ORKNEY REGION}

The Orkney region was used a case study. Due to its large energetic potential (Simon P. Neill, Reza
Hashemi, \& Matt J. Lewis 2014, Peter E. Robins, Simon P. Neill, Matt J. Lewis, \& Sophie L. Ward 2015), the European Marine Energy Centre (EMEC) has set up several test sites, providing the opportunity to test full-scale grid-connected prototype devices for both wave and tidal conditions. Regarding tidal stream energy, two test sites are available: (i) the Fall of Warness (Figure 1) a grid-connected facility, which offers five test berths at depths ranging from $25 \mathrm{~m}$ to $50 \mathrm{~m}$ in an area of approximately $8 \mathrm{~km}^{2}$ and (ii) the scale tidal test site at Shapinsay Sound (Figure 1), a non-grid connected tests site, which provides TEC developers with the opportunity to test their prototypes in real sea conditions. Although detailed studies assessing the tidal stream resource have been conducted in the region (Peter E. Robins, Simon P. Neill, Matt J. Lewis, \& Sophie L. Ward 2015), none of them has been carried out following the IEC-62600-201 methodology. For all the above mentioned reasons, this region appears as an excellent location to evaluate the main aspects related to the numerical modelling of IEC-62600-201.

\section{DELFT3D-FLOW NUMERICAL MODEL}

With the aim of determining the tidal stream energy resource in the area of study, the open source hydrodynamic model Delft3D-Flow was used (Hydraulics, Delft. 2006).

\subsection{Governing equations}

Delft3D-Flow is a finite difference code, which solves the unsteady shallow water equations in two (depth-averaged) or three dimensions. The system of equations consists of the horizontal and vertical

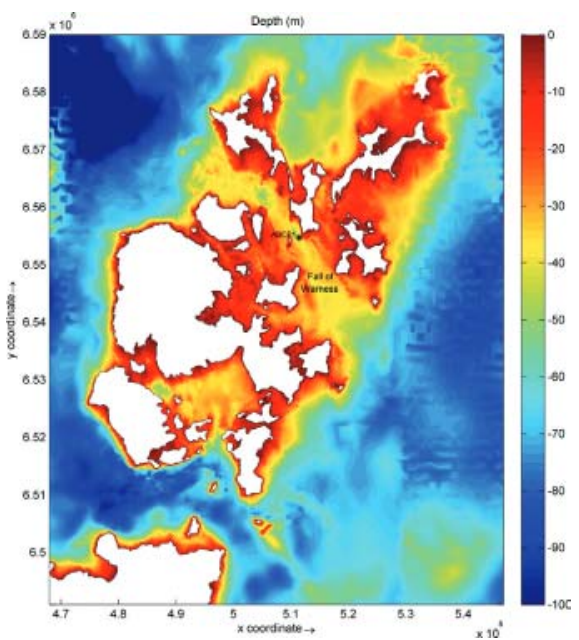

Figure 1. Case of study: Orkney region. 
equations of motion, the continuity equation, and the transport equations for conservative constituents. The flow is forced by the tide at the open boundaries, wind stress at the free surface and pressure gradients due to free surface gradients (barotropic) or density gradients (baroclinic). Source and sink terms are included in the equations to model the discharge and withdrawal of water. Thus, the main equations solved by the model are:

i. The continuity equation:

$$
\frac{\partial u}{\partial x}+\frac{\partial v}{\partial y}+\frac{\partial w}{\partial z}=Q
$$

where, $x, y$ and $z$ represent the east, north and vertical axes, respectively; $u, v$ and $w$ are the velocity components on the $x, y$ and $z$ directions, respectively; and $Q$ represents the intensity of mass sources per unit area. ii. The momentum equations in the horizontal direction:

$$
\begin{aligned}
\frac{D u}{D t}= & f v-g \frac{\partial \zeta}{\partial x}-\frac{g}{\rho_{0}} \int_{z^{\prime}=z}^{z^{\prime}=\zeta} \frac{\partial \rho}{\partial x} d z^{\prime} \\
& +v_{h}\left(\frac{\partial^{2} u}{\partial x^{2}}+\frac{\partial^{2} u}{\partial y^{2}}\right)+v_{v}\left(\frac{\partial^{2} u}{\partial z^{2}}\right), \\
\frac{D v}{D t}= & -f u-g \frac{\partial \zeta}{\partial y}-\frac{g}{\rho_{0}} \int_{z^{\prime}=z}^{z^{\prime}=\zeta} \frac{\partial \rho}{\partial y} d z^{\prime} \\
& +v_{h}\left(\frac{\partial^{2} v}{\partial x^{2}}+\frac{\partial^{2} v}{\partial y^{2}}\right)+v_{v}\left(\frac{\partial^{2} v}{\partial z^{2}}\right),
\end{aligned}
$$

where, $\zeta$ stands for the free surface elevation relative to a reference plane $(z=0), g$ is the gravitational acceleration, $\rho$ and $\rho_{o}$ are the density and reference density of sea water, respectively; $f$ is the Coriolis parameter and $v_{h}$ and $v_{v}$ are the horizontal and vertical eddy viscosity coefficients, respectively.

iii. The momentum equation in the vertical direction:

Under the shallow-water assumption, the conservation of momentum in the vertical direction is simplified to the hydrostatic pressure, $p$, distribution:

$$
\frac{\partial p}{\partial z}=-\rho g,
$$

iv. The transport equation:

$$
\frac{D c}{D t}=D_{h}\left(\frac{\partial^{2} c}{\partial x^{2}}+\frac{\partial^{2} c}{\partial y^{2}}\right)+D_{\nu} \frac{\partial^{2} c}{\partial z^{2}}-\lambda_{d} C+R_{s},
$$

where, $c$ represents either salinity or temperature, $\lambda_{d}$ represents a first order decay process, $D_{h}$ and $D_{v}$ are the horizontal and vertical eddy diffusivity coefficients, respectively; and, finally, $R_{S}$ is the source term per unit area.

\subsection{TEC modelling in Delft3D-Flow}

In order to take into account the potential impacts on the total energy production of a tidal array due to the local alterations on the hydrodynamics (Ramos, Carballo, Sanchez, Veigas, \& Iglesias 2014), the effects related to the operation of the TECs must be simulated. Despite the large number of methods used to model the operation of a TEC within large-scale hydrodynamic codes such as Delft3D-Flow, Telemac, FVCOM and MIKE, the momentum sink approach appears as one of the most popular (Sanchez, Carballo, Ramos, \& Iglesias 2014). Consistent with the momentum sink approach, Delft3D-Flow presents the so-called "Porous Plate" tool (Hydraulics, Delft. 2006), which can be used to simulate the operation of a TEC (Sanchez, Carballo, Ramos, \& Iglesias 2014) by adding two momentum sink terms $\left(M_{x}, M_{y}\right)$ into the right-hand side of the momentum equations (Eq. 3 and 4), which account for the loss of momentum of the flow due to the presence of the TECs. The added momentum terms can be expressed as (Hydraulics, Delft. 2006):

$$
\begin{aligned}
& M_{x}=-c_{\text {loss }-x} \frac{u^{2}}{\Delta x}, \\
& M_{x}=-c_{\text {loss }-y} \frac{v^{2}}{\Delta y},
\end{aligned}
$$

where the coefficients $c_{\text {loss }-x}$ and $c_{\text {loss }-y}$ must be defined during the setup process of the model. In this case, the methodology defined in (Baston, Waldman, \& Side 2015, Waldman S. 2016), which relates the $c_{\text {loss }}$ coefficients with the thrust coefficient $(C)$ exerted by a TEC was used. Consequently, $c_{\text {loss }}$ coefficients can be defined as:

$$
\begin{aligned}
& c_{\text {loss }-x}=\frac{-2 \gamma_{x}}{\left(1+\sqrt{1-\gamma_{x}}\right)^{2}}, \\
& c_{\text {lass }-y}=\frac{-2 \gamma_{y}}{\left(1+\sqrt{1-\gamma_{y}}\right)^{2}},
\end{aligned}
$$

with

$$
\begin{aligned}
& \gamma_{x}=\frac{C_{t} A_{t} \sin \theta}{n \Delta y \Delta z}, \\
& \gamma_{y}=\frac{C_{t} A_{t} \cos \theta}{n \Delta x \Delta z},
\end{aligned}
$$

where $A_{t}$ is the total area occupied by the TEC, $\theta$ is the angle between the $x$ direction and the TEC axis (Figure 2), $n$ is the number of vertical layers of the model occupied by the TEC, $C_{t}$ is the thrust coefficient of the TEC, $\Delta x$ and $\Delta y$ are the grid sizes in the $x$ 


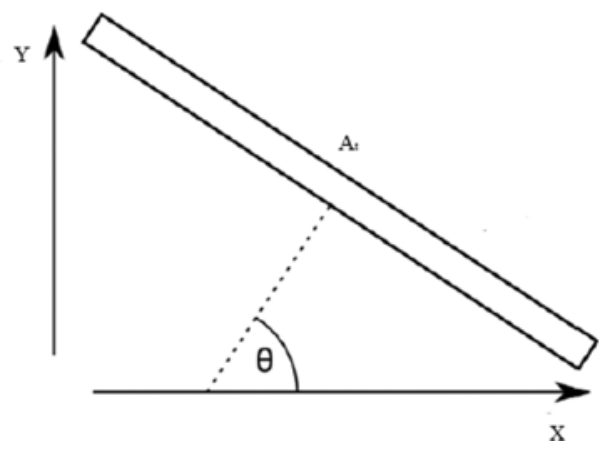

Figure 2. Schematic representation of a TEC for Delft3D implementation.

Table 3. Main characteristics of the Evopod Turbine.

Evopod Turbine

\begin{tabular}{ll}
\hline Diameter $(m)$ & 20 \\
Cut-in velocity $\left(m s^{-1}\right)$ & 0.7 \\
Cut-off velocity $\left(m s^{-1}\right)$ & 4.4 \\
Rated velocity days $\left(m s^{-1}\right)$ & 3.15 \\
Rated power $(\mathrm{kW})$ & 1680
\end{tabular}

and $y$ directions, respectively. Finally, it is important to point out that this approach presents some limitations related to the discretisation of the TEC, which are highlighted in (Baston, Waldman, \& Side 2015).

On these grounds, the Evopod Turbine (OceanFlowEnergy 2010), a floating horizontal axis tidal stream turbine, was used as a reference TEC for the present study. The main characteristics of the Evopod turbine are summarised in Table 3.

\section{MODEL IMPLEMENTATION}

Four different hydrodynamic models were implemented in the area of study for the two stages proposed by the IEC-62600-201. With regard to Stage 1, three models with the notation of S1_500, S1_250 and S1_125 were set up while, for Stage 2, a model with the notation of S2 25 was implemented. A detailed description, regarding the implementation of the models, is presented in the following sections.

\subsection{Modelling area}

Regarding the computational domain, Stage 1 models (S1_500, S1_250 and S1_125) were implemented in 2D (i.e. depth-averaged) using Cartesian grids. Three different mesh sizes were used, $500 \times 500 \mathrm{~m}, 250 \times 250 \mathrm{~m}$ and $125 \times 125 \mathrm{~m}$ (Figure 3), respectively; with the aim of assessing the sensitivity of the grid size in terms of both accuracy and computational effort. Conversely, S2_25

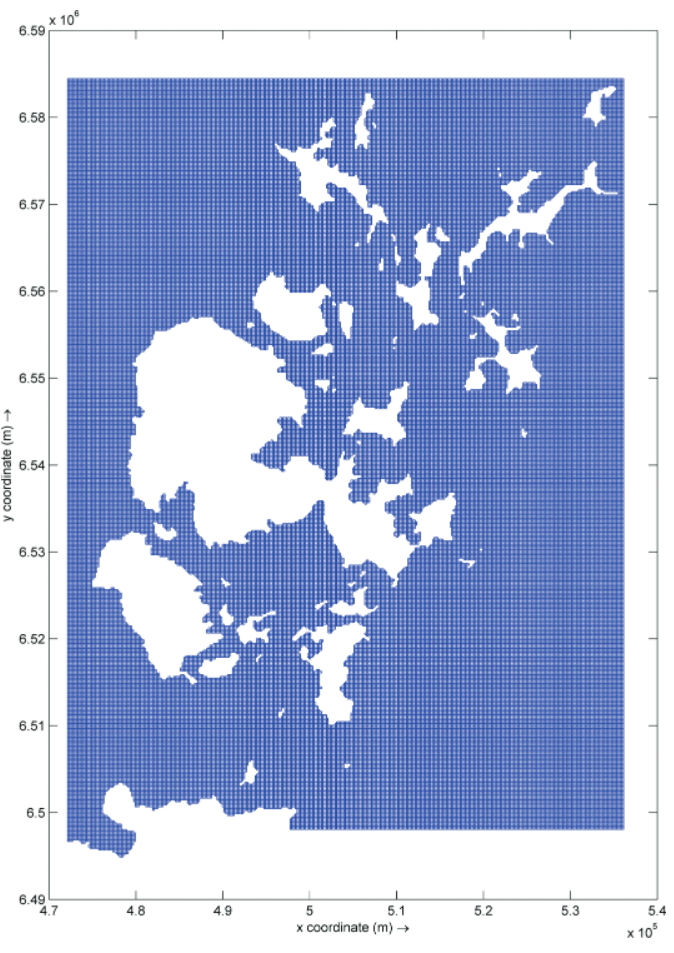

Figure 3. S1_125 computational grid.

(i.e. layout design) was implemented in 3D using the $\sigma$-layer approach for the vertical discretisation. In this case, a uniform distribution of five $\sigma$-layers was prescribed, using a homogeneous thickness for all the layers corresponding with $20 \%$ of the local water depth. Regarding the horizontal discretisation, a Cartesian grid was also used, covering the area corresponding with the tidal test site at the Fall of Warness (Figure 1), with a constant grid size of $25 \times 25 \mathrm{~m}$, which allows for a much more accurate representation of the shorelines and bathymetry.

Finally, the bathymetric data for the region was obtained from the British Oceanographic Data Center (BODC) through the bathymetric data sets contained in the General Bathymetric Chart of the Oeans (GEBCO), which were interpolated onto the computational domains of the models by means of the Delft3D-QUICKIN toolbox.

\subsection{Forcing factors and boundary conditions}

With respect to the forcing factors of the models, only astronomical and Coriolis forcing were included in the model set-up. For the boundary conditions of the models, two different approaches were used. For models S1_500 and S1_250, along the ocean boundary a Dirichlet boundary condition was imposed, i.e. the sea level was prescribed as a function of time using the major tidal harmonics (M2, S2, N2, K2, K1, O1, P1, Q1, MF, MM, M4, MS4, MN4) 
obtained from the database TPXO 7.2, a global model of ocean tides that solves the Laplace equations using data from tide gauges and the TOPEX/ Poseidon Satellite (Dushaw, Egbert, Worcester, Cornuelle, Howe, \& Metzger 1997). On the other hand, for models S1_125 and S2_25, the boundary conditions were obtained by means of a nesting approach. Therefore, models S1_125 and S2_25 were nested inside model S1_250 with the aim of generating time-series of the free surface elevation alongside the ocean boundaries of the nested models. Nesting has many benefits, including a better and more realistic boundary conditions provided for the fine resolution model. In addition, the boundary conditions at the land margins were set to null velocity and free slip (zero shear stress). At the sea-bed, the shear stress is computed by using a quadratic stress law:

$$
\overline{\tau_{b}}=\frac{\rho_{0} g\left|\overline{u_{b}}\right| \overline{u_{b}}}{C_{3 D}^{2}},
$$

where $\overrightarrow{u_{b}}$ is the horizontal velocity in the bottom layer and $C_{3 D}$ is the 3D-Chezy coefficient, which is calculated from the two-dimensional Chezy coefficient, $C_{2 D}$, as follows:

$C_{3 D}=C_{2 D}+\frac{\sqrt{g}}{\varkappa} \ln \frac{\Delta z_{b}}{2 H}$,

where $\Delta z_{b}$ represents the vertical distance from the seabed to the nearest computational grid point, $\varkappa$ is the von Karman constant $(\varkappa=0.41)$ and $H$ is the total water depth. Finally, $C_{2 D}$, is computed by means of the Manning coefficient, $n$ :

$C_{2 D}=\frac{\sqrt[6]{H}}{n}$

and the wind stress exerted on the free surface is computed as follows:

$$
\bar{\tau}_{s}=C_{d} \rho_{a}\left|\vec{U}_{10}\right| \vec{U}_{10},
$$

where $\vec{U}_{10}$ represents the wind velocity vector at $10 m$ height above the sea surface, $\rho_{a}$ is the air density, and $C_{d}$ is a dimensionless drag coefficient. According to Smith (Smith 1980) for wind velocities below $6 \mathrm{~ms}^{-1}$.

$$
C_{d}=1.1 \cdot 10^{-3} \text {, }
$$

while, according to Yelland (Yelland, Moat, Taylor, Pascal, Hutchings, \& Cornell 1998) for wind velocities over $6 \mathrm{~ms}^{-1}$ :

$$
C_{d}=\left(0.50+0.0071 U_{10}\right) \cdot 10^{-3},
$$

\section{RESULTS}

\subsection{Model validation}

In order to ensure that the models accurately predict the hydrodynamic conditions of the area of study, they were validated by comparing the numerical results and measured data of the flow velocity. The field data were gathered by means of an Acoustic Doppler Current Profiler (ADCP), deployed by the European Marine Energy Center (EMEC) at the Fall of Farness tidal test site (Figure 1), covering the period from 20 April 2005 to 4 May 2005.

For the models corresponding to Stage 1 (S1_500, S1_250, S1_125), a good agreement was found between the time-series of the computed and measured magnitude of the flow velocity (Figure 4), with only minor differences in the peak velocities in some tidal cycles. The corresponding statistical analysis is summarised in Table 4. As expected, the results of the validation improved with the degree of refinement of the models (i.e. higher grid resolution).

Regarding the Stage 2 model (S2_25), a validation at different levels of the water column was carried out in order to ensure that the model accurately solves the 3D behaviour of the flow. Therefore, a comparison between the time-series of the computed and measured values of the flow velocity at the surface, mid-depth and bottom layers was carried out (Figure 5). The agreement in general is very good, with only minor differences in the maximum velocities of some tidal cycles, and again, it shows an improvement in comparison with Stage 1 models (Table 4), which appears to validate the IEC-62600-201 protocol.

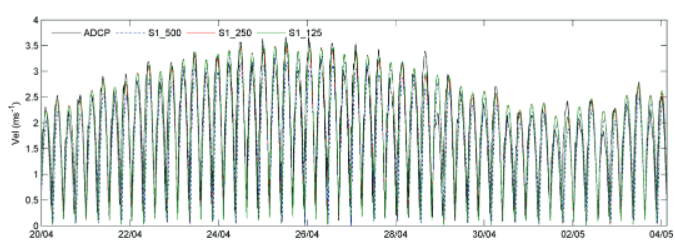

Figure 4. Stage 1 model validation.

Table 4. Root Mean Square Error (RMSE) and Correlation coefficients $(\mathrm{R})$ of the model validation.

\begin{tabular}{lll}
\hline & RMSE $\left(m^{-1}\right)$ & $\mathrm{R}$ \\
\hline S1_500 & 0.5699 & 0.7961 \\
S1_250 & 0.3912 & 0.9051 \\
S1_125 & 0.3903 & 0.9074 \\
S2_25 Surface Layer & 0.3935 & 0.9185 \\
S2_25 Middle Layer & 0.3982 & 0.9195 \\
S2_25 Bottom Layer & 0.3952 & 0.9166 \\
\hline
\end{tabular}




\subsection{TEC Annual Energy Production (TAEP)}

As mentioned in Section 2, the main goal of the different modelling stages proposed by IEC-62600201 is to determine the TAEP in a certain location. Accordingly, models S1_500, S1_250, S1_125, S2 25 were used to estimate the TAEP and illustrate the variations among the different models in terms of energy estimation. For this purpose, the area corresponding to the location of the ADCP was used as case study (Figure 1), while the Evopod Turbine was used as a reference TEC (Section 4.2). Finally, the models were run for an average year with the aim of obtaining the annual velocity distribution (Figure 6) and the annual exceedance probability (Figure 7).

Overall, it can be observed that the area of study presents a remarkable resource with the presence of tidal peak velocities exceeding $4 \mathrm{~ms}^{-1}$, and with probabilities of exceedance in the order of 0.5 for tidal velocities larger than $2.0 \mathrm{~ms}^{-1}$. In addition, it can be observed that there are remarkable differences among the results offered by the different models. For instance, models S1_500 and S1_250 appear not to be able to properly estimate the exceedance probability for flow velocities higher than $2.0 \mathrm{~ms}^{-1}$, which may translate into an underestimation of the TAEP. Conversely, models S1_125 and S2_25 present a very similar behavior for both the annual velocity distribution and the annual exceedance probability.

Finally, the TAEP was computed for the different models following the procedure proposed by IEC-62600-201 (Eq. 1). As expected, the results obtained show that models S1 500 and S1_250 underestimate the TAEP in comparison with models S1_125 and S2_25 (Table 5). This translates into differences up to $19.10 \%$ in comparison with S2_25, which was used as a reference, since it offers the best validation results.
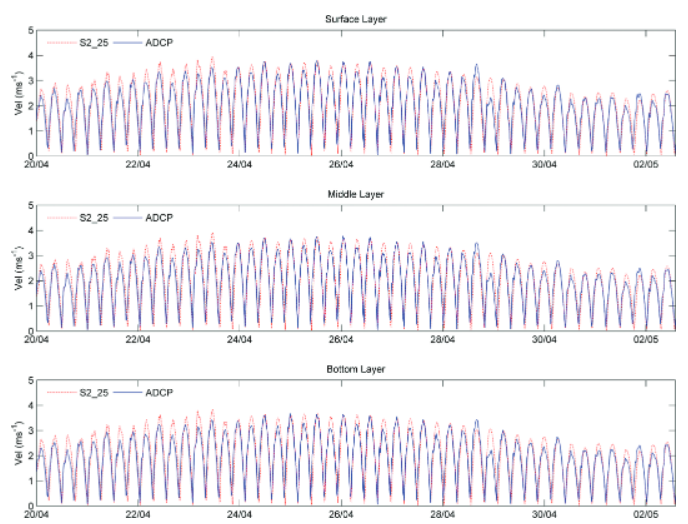

Figure 5. Stage 2 model validation.

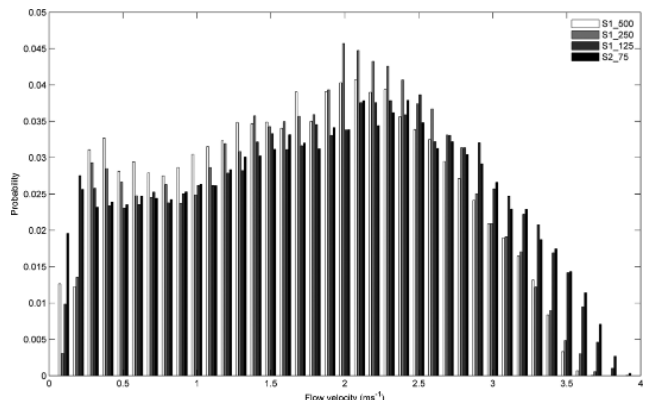

Figure 6. Annual velocity distribution computed from the models.

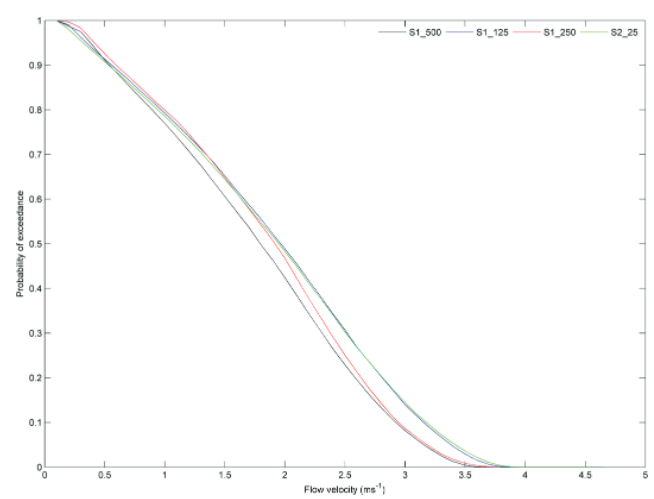

Figure 7. Annual exceedance probability computed from the models.

Table 5. TAEP and differences among the different models.

\begin{tabular}{lll}
\hline & TAEP $(G W h)$ & \% Dif-S2_25 \\
\hline S1_500 & 3.81 & 19.10 \\
S1_250 & 4.11 & 10.26 \\
S1_125 & 4.56 & 0.56 \\
S2_25 & 4.53 & - \\
\hline
\end{tabular}

\subsection{Effects of TEC operation in TAEP estimation}

With the purpose of validating the methodology proposed by IEC-62600-201 regarding the necessity of including the TEC operation in the hydrodynamic modelling for tidal projects with a capacity larger than $10 \mathrm{MW}$ and/or the $2 \%$ of the theoretical energy resource due to potential disturbances on the available tidal resource, a tidal farm of 7 Evopod Turbines with an installed power capacity of $11.76 \mathrm{MW}$ was proposed in the Fall of Warness tidal test site (Figure 1). This area presents an outstanding tidal resource, with homogeneous flow velocities exceeding $3.5 \mathrm{~ms}^{-1}$ and $2.2 \mathrm{~ms}^{-1}$ at mid-ebb and mid-flood of a mean-sprig tide, respectively (Figure 9). Following (Ramos, 


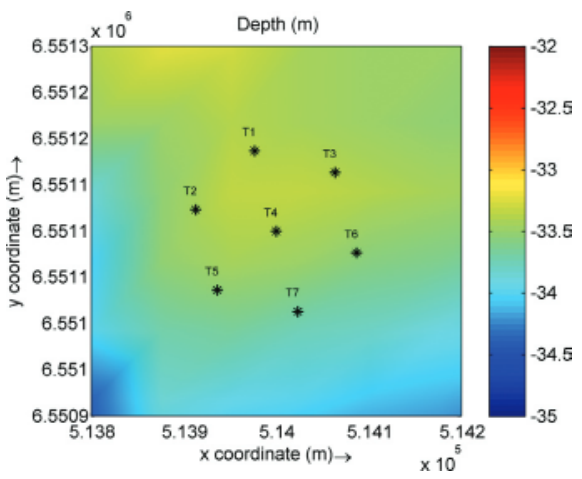

Figure 8. Location and layout of the proposed tidal farm.
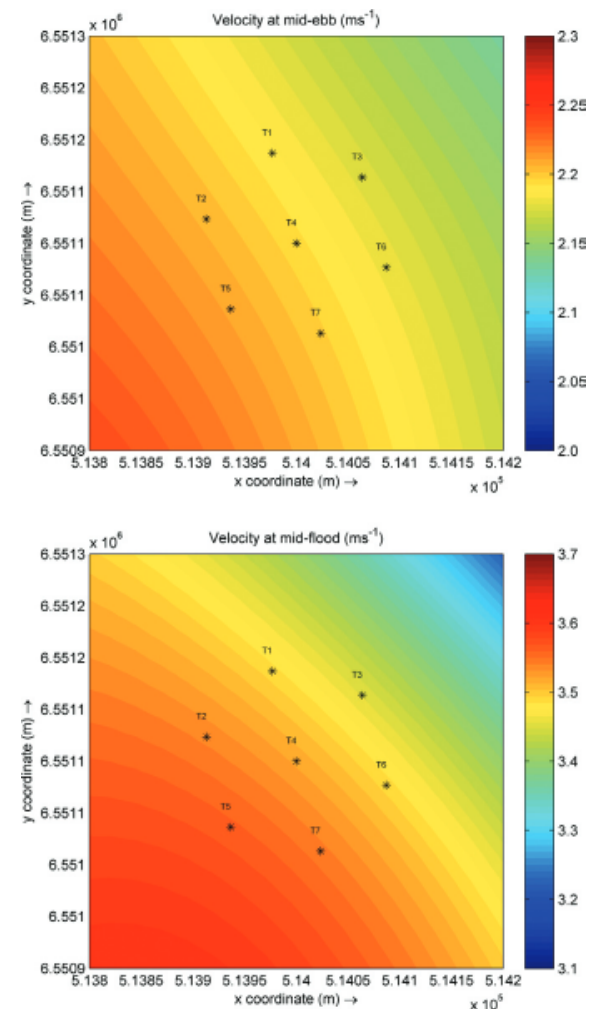

Figure 9. Mid-ebb and mid-flood velocities at the proposed tidal farm.

Carballo, Sanchez, Veigas, \& Iglesias 2014), a triangular distribution for the tidal turbines was chosen, with the rows separated $100 \mathrm{~m}$ (five-diameter) from each other (in the NW-SE direction) and a lateral separation of $60 \mathrm{~m}$ (three-diameter) among the turbines of the same row (in the SW-NE direction) (Figure 8).
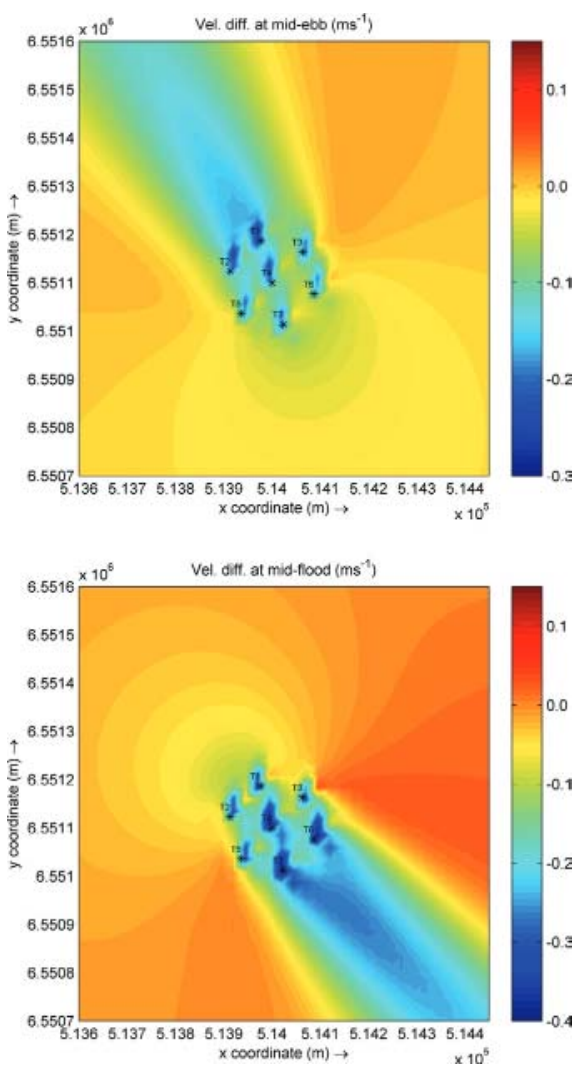

Figure 10. Veloctiy differences at Mid-ebb and midflood between the baseline and energy extraction cases.

Table 6. Energy production of each individual turbine for the baseline and energy extraction cases.

\begin{tabular}{llll}
\hline & $\begin{array}{l}\text { Baseline case } \\
\text { Energy prod. } \\
(M W h)\end{array}$ & $\begin{array}{l}\text { Energy extraction case } \\
\text { Energy prod. } \\
(M W h)\end{array}$ & $\begin{array}{l}\text { Diff. cases } \\
\text { Base-Energy } \\
\text { ext. }(\%)\end{array}$ \\
\hline T1 94.88 & 84.25 & 12.63 \\
T2 & 97.77 & 89.05 & 9.79 \\
T3 & 90.90 & 83.64 & 8.68 \\
T4 & 95.61 & 84.77 & 12.79 \\
T5 & 98.70 & 93.64 & 5.40 \\
T6 & 92.23 & 83.32 & 10.69 \\
T7 96.76 & 86.80 & 11.48 \\
\hline
\end{tabular}

Once the location and configuration of the tidal farm were defined, the S2_25 model was run with and without considering the operation of the tidal turbines, energy extraction and baseline cases, respectively; for a period corresponding with a complete spring-neap tidal cycle (i.e. 14.8 days). The operation of the Evopod turbines in Delft3D was modelled according to the methodology presented in Section 4.2 , considering a constant value of the thrust 
coefficient $C_{t}$ of 0.85 (Baston, Waldman, \& Side 2015). The effects on the tidal flow (magnitude and direction) were investigated by computing the differences in the velocities between the energy extraction and baseline cases. Figure 10 shows the differences in the tidal flow between the energy extraction and baseline cases at mid-ebb and mid-flood of a mean spring tide. Overall, it can be observed that there is a velocity reduction upstream and especially downstream (up to $0.3 \mathrm{~ms}^{-1}$ ) of the tidal farm, which extends hundred of meters from the tidal farm. With respect to each individual turbine, a similar pattern can be observed with the additional feature that the flow is diverted and concentrated at both sides of the turbines resulting in an increase of the tidal velocities (up to $0.1 \mathrm{~ms}^{-1}$ ). Finally, in order to quantify these alterations in terms of power production, the energy generated for each individual turbine during the spring-neap tidal cycle was computed for the baseline and energy extraction cases, respectively. With reference to Table 6 , the layout proposed for the tidal farm results in a destructive effect (i.e. reduction of the power production); since every single turbine shows a reduction in the generated energy, which ranges from $5.40 \%$ (T5) to $12.63 \%$ (T1).

\section{DISCUSSION}

This section aims to offer some feedback to the IEC62600-201, with respect to the main aspects tackled in this investigation: the model setup process and the methodology proposed for the quantification of the TEC Annual Energy Production (TAEP).

Overall, the methodology proposed by the IEC62600-201 for the modelling setup process appears to perform well for the present case study, proving that the increase in the degree of refinement of the different model classes (Stages 1 and 2) reduces the level of uncertainty in the estimation of the tidal flow and, therefore, in the TAEP quantification. However, the results obtained in the present study may also offer some interesting insight into the model setup process. The results in terms of accuracy improved dramatically from S1_500 to S1_250 (grid resolution of $500 \times 500 \mathrm{~m}$ vs $250 \times 250 \mathrm{~m}$ ); however, from S1_250 to S1_125 and S2_25 (grid resolutions of $125 \times 125 \mathrm{~m}$ and $25 \times 25 \mathrm{~m}$, respectively) the improvement felt is almost negligible, especially in the case of S1_125 and S2_25. On the other hand, the computational effort increased considerably as the grid resolution was finer, especially for the case of S2_25. This fact should not be considered of minor importance, especially to find the right balance between the accuracy and the computational effort required by the model, since high-resolution models, such as S2_25 or S1_125, require higher computational times, whereas the level of accuracy provided is only slightly better than S1_250. Therefore, taking all these apects into consideration, the requirements for the Stage 2 model setup regarding the minimum grid resolution could be revisited for future editions of the IEC-62600-201.

With respect to the methodology developed by IEC-62600-201 for the TAEP quantification, the results obtained for the present work prove that the methodology proposed is robust and coherent. However, there are some issues that must be addressed in more detail, especially those related to the modelling of the TEC operation within a hydrodynamic model. The results obtained in the present case study show indeed that there is an overestimation of TAEP (up to $12 \%$ ) for the case in which the model was run without taking into account the TEC operation and, therefore, it should be taken into account for large tidal energy projects. Nevertheless, when modelling the operation of a TEC within a hydrodynamic model different methodologies can be found in the literature, with most presenting significant limitations. In the present study, the momentum sink approach was used in Delft3D via the so-called Porous plate tool, whose main limitation is the assumption of a constant thrust coefficient $\left(C_{t}\right)$ to cover the complete turbine operation. Therefore, taking all these facts into consideration, the IEC-62600-201 should clearly state which of the approaches present in the literature should be used for modelling the TEC operation, or to develop its own standard methodology, with the aim of avoiding misrepresentations in the TAEP estimation, which may jeopardise the initial viability of tidal stream projects.

Finally, it is important to point out that all these recommendations are based on the results obtained for the present study and, therefore, they should be supported by other works. For this purpose, more studies like the present one may be carried out for different locations subject to different tidal ranges and conditions with the purpose of offering to IEC-62600-201 a broader feedback for future editions. So far, only a few tidal resource assessments based on IEC-62600-201 were found in the literature (Cornett, Baker, Toupin, Piche, \& Nistor 2014, Cornett, Toupin, \& Nistor 2015).

\section{CONCLUSIONS}

In recent years, the interest in harvesting the tidal stream energy resource has translated into a large number of tidal stream resource assessments. However, different approaches were used regarding different and relevant aspects such as time scales, effects derived from the wave-current interactions and the methodologies used for the representation of TEC operation. Consequently, the IEC has developed a methodology (IEC-62600-201) with the aim of standardising tidal stream resource 
characterisation. Therefore, the aim of this work is to explore the utility of the IEC-62600-201 by means of a case study, focusing on the main aspects of the modelling setup process and the TAEP estimation.

Overall, the IEC-62600-201 has proven to be a robust and coherent methodology, which offers a set of recommendations and rules to carry out a precise tidal stream resource characterisation. Regarding the modelling setup process, the results obtained show that the grid resolution plays a limited role on the accuracy of the models. In this context, the minimum grid resolution required for the Stage 2 models could be increased for future editions of the standard. From the point of view of the TAEP estimation, the main issue that may be addressed for future editions of the IEC-62600201 is to define a standard methodology to simulate the TEC operation within a hydrodynamic model, which is essential to obtain an accurate representation of the behavior of the tidal flow in the vicinity of a tidal farm and, therefore, a good estimation of the TAEP. Finally, it is important to point out that these recommendations should be corroborated with the feedback from other works of the same nature as the present one.

In summary, this work explores the main characteristics of the IEC-62600-201 offering some feedback for future editions, although some important aspects such as the wave-current interaction are outside the scope of this work and will be dealt with as a continuation of this research

\section{ACKNOWLEDGMENT}

This research is based upon works supported by Science Foundation of Ireland under Grant No. $12 / \mathrm{RC} / 2302$ for the Marine Renewable Ireland (MAREI) centre. During this work, V. Ramos was supported by the I2C postdoctoral grant ED481B 2014/059-0 (Plan Galego de Investigacion Innovacion e Crecemento 2011-2015) of the Xunta de Galicia (Spain). Its authors are also indebted to the European Marine Energy Centre (EMEC) for its contribution with the ADCP data and the Irish Centre for High-End Computing (ICHEC) for its cooperation in the computational tasks.

\section{REFERENCES}

(2004, 12). http://www.emec.org.uk/.

(2014). Iec-ts 62600-201: Marine energy wave, tidal and other water current converters part 201: Tidal energy resource assessment and characterisation.

Bahaj, A.S. (2011). Generating electricity from the oceans. Renewable and Sustainable Energy Reviews 15(7), 3399-3416.

Baston, S., S. Waldman, \& J. Side (2015). Modelling energy extraction in tidal flows. MASTS Position Paper. 1.
Carballo, R., G. Iglesias, \& A. Castro (2009). Numerical model evaluation of tidal stream energy resources in the Ria de Muros (NW Spain). Renewable Energy 34(6), 1517-1524.

Cornett, A., S. Baker, M. Toupin, S. Piche, \& I. Nistor (International Conference on Ocean Energy (ICOE), November 4-6, 2014). Appraisal of IEC Standards for Wave and Tidal Energy Resource Assessment.

Cornett, A., M. Toupin, \& I. Nistor (2015). Appraisal of iec technical specification for tidal energy resource assessment at minas passage, bay of fundy, canada. In Proceedings of the 11th European Wave and Tidal Energy Conference.

Dushaw, B.D., G.D. Egbert, P.F. Worcester, B.D. Cornuelle, B. M. Howe, \& K. Metzger (1997). A topex/ poseidon global tidal model (tpxo.2) and barotropic tidal currents determined from long-range acoustic transmissions. Progress in Oceanography 40, 337-367. Tidal Science In Honour of David E. Cartwright.

Fergal O. Rourke, Fergal Boyle, \& Anthony Reynolds (2010). Tidal energy update 2009. Applied Energy 87(2), 398-409.

Hashemi, M. R., S. P. Neill, P. E. Robins, A. G. Davies, \& M.J. Lewis (2015). Effect of waves on the tidal energy resource at a planned tidal stream array. Renewable Energy 75, 626-639.

Hydraulics, Delft. (2006). Delft3D-Flow user manual. Delft, the Netherlands.: Hydraulics, Delft.

Jinhai Zheng, Peng Dai, \& Jisheng Zhang (2015). Tidal stream energy in china. Procedia Engineering 116, 880-887. 8th International Conference on Asian and Pacific Coasts (APAC 2015).

OceanFlowEnergy $(2010,12)$. http://www.oceanflowenergy.com/.

Peter E. Robins, Simon P. Neill, Matt J. Lewis, \& Sophie L. Ward (2015). Characterising the spatial and temporal variability of the tidal-stream energy resource over the northwest European shelf seas. Applied Energy $147,510-522$

Ramos, V., R. Carballo, M. Sanchez, M. Veigas, \& G. Iglesias (2014). Tidal stream energy impacts on estuarine circulation. Energy Conversion and Management 80(0), 137-149.

Rourke, F.O., F. Boyle, \& A. Reynolds (2009). Renewable energy resources and technologies applicable to Ireland. Renewable and Sustainable Energy Reviews 13(8), 1975-1984.

Sanchez, M., R. Carballo, V. Ramos, \& G. Iglesias (2014). Floating vs. bottom-fixed turbines for tidal stream energy: A comparative impact assessment. Energy 72, 691-701.

Simon P. Neill, M. Reza Hashemi, \& Matt J. Lewis (2014). The role of tidal asymmetry in characterizing the tidal energy resource of Orkney. Renewable Energy $68,337-350$.

Smith, S.D. (1980). Wind stress and heat flux over the ocean in gale force winds. Journal of Physical Oceanography 10(5), 709-726.

Waldman S, Baston S, N.R.C.A.V.V.S.J. (2016). Implementation of tidal turbines in MIKE 3 and Delft3D models of Pentland Firth \& Orkney Waters.

Yelland, M., B. Moat, P. Taylor, R. Pascal, J. Hutchings, \& V. Cornell (1998). Wind stress measurements from the open ocean corrected for airflow distortion by the ship. Journal of Physical Oceanography 28(7), 1511-1526. 